\title{
On the Janowski convexity and starlikeness of the confluent hypergeometric function
}

\author{
Rosihan M. Ali Saiful R. Mondal V. Ravichandran
}

\begin{abstract}
For $-1 \leq B<A \leq 1$, conditions on $A, B, a, c$ are determined that ensure the confluent hypergeometric function $\Phi(a ; c ; z)$ satisfies the subordination $\Phi(a ; c ; z) \prec(1+A z) /(1+B z)$. This gives rise to conditions for $(c / a)(\Phi(a ; c ; z)-1)$ to be close-to-convex, $\Phi(a ; c ; z)$ to be Janowski convex, and $z \Phi(a ; c ; z)$ to be Janowski starlike.
\end{abstract}

\section{Introduction}

Let $\mathcal{A}$ denote the class of analytic functions $f$ defined in the open unit disk $\mathbb{D}=\{z:|z|<1\}$ normalized by the conditions $f(0)=0=f^{\prime}(0)-1$. If $f$ and $g$ are analytic in $\mathbb{D}$, then $f$ is subordinate to $g$, written $f(z) \prec g(z)$, if there is an analytic self-map $w$ of $\mathbb{D}$ satisfying $w(0)=0$ and $f(z)=g(w(z))$. For $-1 \leq B<A \leq 1$, let $\mathcal{P}[A, B]$ be the class consisting of normalized analytic functions $p(z)=1+c_{1} z+\cdots$ in $\mathbb{D}$ satisfying

$$
p(z) \prec \frac{1+A z}{1+B z}
$$

For instance, if $0 \leq \beta<1$, then $\mathcal{P}[1-2 \beta,-1]$ is the class of functions $p(z)=1+c_{1} z+\cdots$ satisfying $\operatorname{Re} p(z)>\beta$ in $\mathbb{D}$.

Received by the editors in May 2013 - In revised form in September 2014.

Communicated by $\mathrm{H}$. De Schepper.

2010 Mathematics Subject Classification : 30C45, 30C80, 40G05.

Key words and phrases: Subordination, Janowski starlike and convex functions, confluent hypergeometric function. 

ing

The class $\mathcal{S}^{*}[A, B]$ of Janowski starlike functions [6] consists of $f \in \mathcal{A}$ satisfy-

$$
\frac{z f^{\prime}(z)}{f(z)} \in \mathcal{P}[A, B]
$$

For $0 \leq \beta<1, \mathcal{S}^{*}[1-2 \beta,-1]:=\mathcal{S}^{*}(\beta)$ is the usual class of starlike functions of order $\beta ; \mathcal{S}^{*}[1-\beta, 0]:=\mathcal{S}_{\beta}^{*}=\left\{f \in \mathcal{A}:\left|z f^{\prime}(z) / f(z)-1\right|<1-\beta\right\}$, and $\mathcal{S}^{*}[\beta,-\beta]:=\mathcal{S}^{*}[\beta]=\left\{f \in \mathcal{A}:\left|z f^{\prime}(z) / f(z)-1\right|<\beta\left|z f^{\prime}(z) / f(z)+1\right|\right\}$. These classes have been studied, for example, in [2,3]. A function $f \in \mathcal{A}$ is said to be close-to-convex of order $\beta[5,9]$ if $\operatorname{Re}\left(z f^{\prime}(z) / g(z)\right)>\beta$ for some $g \in \mathcal{S}^{*}:=\mathcal{S}^{*}(0)$.

This paper studies the confluent (Kummer) hypergeometric function $\Phi(a ; c ; z)$ given by

$$
\Phi(a ; c ; z)={ }_{1} F_{1}(a ; c ; z)=\frac{\Gamma(c)}{\Gamma(a)} \sum_{n=0}^{\infty} \frac{\Gamma(a+n)}{\Gamma(c+n)} \frac{z^{n}}{n !}=\sum_{n=0}^{\infty} \frac{(a)_{n}}{(c)_{n}} \frac{z^{n}}{n !},
$$

where $a, c \in \mathbb{C}, c \neq 0,-1,-2, \cdots$, and $(\lambda)_{n}$ denotes the Pochhammer symbol given by $(\lambda)_{0}=1,(\lambda)_{n}=\lambda(\lambda+1)_{n-1}$. The function $\Phi$ is a solution of the differential equation

$$
z \Phi^{\prime \prime}(a ; c ; z)+(c-z) \Phi^{\prime}(a ; c ; z)-a \Phi(a ; c ; z)=0
$$

introduced by Kummer in 1837 [12]. The Kummer hypergeometric function is an entire analytic function in $\mathbb{C}$ and satisfies the relation

$$
c \Phi^{\prime}(a ; c ; z)=a \Phi(a+1 ; c+1 ; z) .
$$

When $\operatorname{Re} c>\operatorname{Re} a>0, \Phi$ can be expressed in the integral form

$$
\Phi(a ; c ; z)=\frac{\Gamma(c)}{\Gamma(a) \Gamma(c-a)} \int_{0}^{1} t^{a-1}(1-t)^{c-a-1} e^{t z} d t .
$$

Several important functions are special cases of the confluent hypergeometric function. These include the Bessel functions, Laguerre polynomials, and the error function. An exposition on $\Phi$ can be found in the book by Temme [12].

Several works $[1,8,10,11]$ have studied geometric properties of the function $\Phi(a ; c ; z)$, which include studies on its close-to-convexity, starlikeness, and convexity. Miller and Mocanu [8] proved that $\operatorname{Re} \Phi(a ; c ; z)>0$ in $\mathbb{D}$ for real $a$ and $c$ satisfying either $a>0$ and $c \geq a$, or $a \leq 0$ and $c \geq 1+\sqrt{1+a^{2}}$. Ponnusamy and Vuorinen [10, Theorem 1.9, p. 77] obtained sufficient conditions for $\operatorname{Re} \Phi(a ; c ; z)>\beta, 0 \leq \beta<1 / 2$. They also determined, as an application of $(1.3)$, conditions that ensure $(c / a)(\Phi(a ; c ; z)-1)$ is close-to-convex of positive order with respect to the identity function. Additionally they derived conditions for close-to-convexity of $z \Phi(a ; c ; z)$ with respect to the starlike function $z /(1-z)$, as well as close-to-convexity of $z \Phi\left(a ; c ; z^{2}\right)$ with respect to the starlike function $z /\left(1-z^{2}\right)$. Constraints on $a$ and $c$ so that $\Phi(a ; c ; z)$ is convex of positive order are also found in [10, Theorem 5.1, p. 88]. For positive $a$, Acharya [1, Theorem 4.1.1, p. 50] proved that $z \Phi(a ; c ; z)$ is starlike and close-to-convex 
with respect to $z$ and $z /(1-z)$ if respectively $2 c \geq 2 a-1+\sqrt{a^{2}+a+1}$ and $6 c^{3}+3 c^{2}(a+6)+3 c\left(-6 a^{2}-3 a+4\right)+a\left(5 a^{2}-221-20\right) \geq 0$.

In Section 2 of this paper, sufficient conditions on $A, B, a, c$ are determined that ensure $\Phi$ satisfies the subordination $\Phi(a ; c ; z) \prec(1+A z) /(1+B z)$. Set in this general framework, our findings are obtained through a computationallyintensive methodology with shrewd manipulations. The benefits of such general results are that they not only extend and improved earlier known works, but by judicious choices of the parameters $A$ and $B$, they give rise to several interesting applications. Examples involving the Laguerre polynomials and the modified Bessel functions are given in Section 3 to illustrate this significance. Sufficient conditions are also obtained for $(c / a) \Phi^{\prime}(a ; c ; z) \in \mathcal{P}[A, B]$, which readily yields conditions for $(c / a)(\Phi(a ; c ; z)-1)$ to be close-to-convex. Section 4 gives emphasis to the investigation of $\Phi(a ; c ; z)$ to be Janowski convex as well as of $z \Phi(a ; c ; z)$ to be Janowski starlike.

The following lemma is needed in the sequel.

Lemma 1.1. $[8,9]$ Let $\Omega \subset \mathbb{C}$, and $\Psi: \mathbb{C}^{3} \times \mathbb{D} \rightarrow \mathbb{C}$ satisfy

$$
\Psi(i \rho, \sigma, \mu+i v ; z) \notin \Omega
$$

whenever $z \in \mathbb{D}, \rho$ real, $\sigma \leq-\left(1+\rho^{2}\right) / 2$ and $\sigma+\mu \leq 0$. If $p$ is analytic in $\mathbb{D}$ with $p(0)=1$, and $\Psi\left(p(z), z p^{\prime}(z), z^{2} p^{\prime \prime}(z) ; z\right) \in \Omega$ for $z \in \mathbb{D}$, then $\operatorname{Re} p(z)>0$ in $\mathbb{D}$.

In the case $\Psi: \mathbb{C}^{2} \times \mathbb{D} \rightarrow \mathbb{C}$, then the condition in Lemma 1.1 reduces to

$$
\Psi(i \rho, \sigma ; z) \notin \Omega,
$$

$\rho$ real and $\sigma \leq-\left(1+\rho^{2}\right) / 2$.

\section{Close-to-convexity of the confluent hypergeometric function}

Here is one main result in a general form that has several interesting ramifications.

Theorem 2.1. Let $-1 \leq B \leq 3-2 \sqrt{2} \approx 0.171573$. Suppose $B<A \leq 1$, and $a$, $c \in \mathbb{R}$ satisfy

$$
c-1 \geq \max \left\{1,\left|1+\frac{(1+B)(1+A)}{A-B} a\right|\right\} .
$$

Further let $A, B, c$ and a satisfy either the inequality

$$
\begin{gathered}
(c-1)^{2}-\left|2(c-1)\left(\frac{a(A+B)}{A-B}+1\right)+\frac{1+B}{1-B}\left(1+\frac{(1+A)(1+B)}{A-B} a\right)\right| \\
+\frac{(c-1)(1+B)}{1-B} \geq \frac{\left(1-A^{2}\right)\left(1-B^{2}\right)}{(A-B)^{2}} a^{2}-2 \frac{A+B}{A-B} a-1
\end{gathered}
$$

whenever

$$
|2(c-1)(1-B)(a(A+B)+A-B)+(1+B)(A-B+(1+A)(1+B) a)|
$$




$$
\geq 2(1-B)\left|A(1+a)^{2}-B(1-a)^{2}\right|,
$$

or the inequality

$$
\begin{aligned}
& \left(2(c-1) \frac{A(1+a)-B(1-a)}{A-B}+\frac{1+B}{1-B}\left(1+\frac{(1+A)(1+B)}{A-B} a\right)\right)^{2} \\
& \leq 4\left(a^{2}+\frac{2(A+B)}{A-B} a+1\right)\left((c-1)^{2}+\frac{(c-1)(1+B)}{1-B}-a^{2} \frac{(1-A B)^{2}}{(A-B)^{2}}\right)
\end{aligned}
$$

whenever

$$
\begin{gathered}
|2(c-1)(1-B)(a(A+B)+A-B)+(1+B)(A-B+(1+A)(1+B) a)| \\
<2(1-B)\left(A(1+a)^{2}-B(1-a)^{2}\right) .
\end{gathered}
$$

If $(1+B) \Phi(a ; c ; z) \neq(1+A)$, then $\Phi(a ; c ; z) \in \mathcal{P}[A, B]$.

Proof. Define the analytic function $p: \mathbb{D} \rightarrow \mathbb{C}$ by

$$
p(z)=-\frac{(1-A)-(1-B) \Phi(z)}{(1+A)-(1+B) \Phi(z)},
$$

where $\Phi(z):=\Phi(a ; c ; z)$. Then

$$
\begin{aligned}
\Phi(z) & =\frac{(1-A)+(1+A) p(z)}{(1-B)+(1+B) p(z)} \\
\Phi^{\prime}(z) & =\frac{2(A-B) p^{\prime}(z)}{((1-B)+(1+B) p(z))^{2}}
\end{aligned}
$$

and

$$
\Phi^{\prime \prime}(z)=\frac{2(A-B)((1-B)+(1+B) p(z)) p^{\prime \prime}(z)-4(1+B)(A-B) p^{\prime 2}(z)}{((1-B)+(1+B) p(z))^{3}} .
$$

Using (2.6)-(2.8), the Kummer differential equation (1.2) yields

$$
\begin{aligned}
z^{2} p^{\prime \prime}(z) & -\frac{2(1+B)}{(1-B)+(1+B) p(z)}\left(z p^{\prime}(z)\right)^{2}+(c-z) z p^{\prime}(z) \\
& -\frac{((1-B)+(1+B) p(z))((1-A)+(1+A) p(z))}{2(A-B)} a z=0
\end{aligned}
$$

With $\Omega=\{0\}$, define $\Psi(r, s, t ; z)$ by

$$
\begin{aligned}
\Psi(r, s, t ; z):=t & -\frac{2(1+B)}{(1-B)+(1+B) r} s^{2}+(c-z) s \\
& -\frac{((1-B)+(1+B) r)((1-A)+(1+A) r)}{2(A-B)} a z .
\end{aligned}
$$


It follows from $(2.9)$ that $\Psi\left(p(z), z p^{\prime}(z), z^{2} p^{\prime \prime}(z) ; z\right) \in \Omega$. To show $\operatorname{Re} p(z)>0$ for $z \in \mathbb{D}$, from Lemma 1.1, it is sufficient to establish $\operatorname{Re} \Psi(i \rho, \sigma, \mu+i v ; z)<0$ in $\mathbb{D}$ for any real $\rho, \sigma \leq-\left(1+\rho^{2}\right) / 2$, and $\sigma+\mu \leq 0$.

With $z=x+i y \in \mathbb{D}$, it readily follows from (2.10) that

$$
\begin{aligned}
\operatorname{Re} \Psi(i \rho, \sigma, \mu+i v ; z)=\mu & -\frac{2\left(1-B^{2}\right)}{(1-B)^{2}+(1+B)^{2} \rho^{2}} \sigma^{2}+(c-x) \sigma+\frac{\rho(1-A B)}{(A-B)} a y \\
& -\frac{(1-B)(1-A)-(1+B)(1+A) \rho^{2}}{2(A-B)} a x .
\end{aligned}
$$

Since $\sigma \leq-\left(1+\rho^{2}\right) / 2$, and $B \in[-1,3-2 \sqrt{2}]$,

$$
\frac{2\left(1-B^{2}\right)}{(1-B)^{2}+(1+B)^{2} \rho^{2}} \sigma^{2} \geq \frac{2\left(1-B^{2}\right)}{(1-B)^{2}+(1+B)^{2} \rho^{2}} \frac{\left(1+\rho^{2}\right)^{2}}{4} \geq \frac{1+B}{2(1-B)}
$$

Thus

$$
\begin{aligned}
\operatorname{Re} \Psi(i \rho, \sigma, \mu+i v ; z) \leq & (\mu+\sigma)+(c-x-1) \sigma+\frac{(1+B)(1+A) \rho^{2}}{2(A-B)} a x \\
& +\frac{\rho(1-A B)}{(A-B)} a y-\frac{(1-B)(1-A)}{2(A-B)} a x-\frac{1+B}{2(1-B)} \\
\leq- & \frac{1}{2}(c-x-1)\left(1+\rho^{2}\right)+\frac{(1+B)(1+A) \rho^{2}}{2(A-B)} a x+\frac{\rho(1-A B)}{(A-B)} a y \\
& \quad-\frac{(1-B)(1-A)}{2(A-B)} a x-\frac{1+B}{2(1-B)} \\
= & p_{1} \rho^{2}+q_{1} \rho+r_{1}:=Q(\rho),
\end{aligned}
$$

where

$$
\begin{aligned}
& p_{1}=-\frac{1}{2}(c-x-1)+\frac{(1+B)(1+A) a x}{2(A-B)} \\
& q_{1}=\frac{1-A B}{A-B} a y, \\
& r_{1}=-\frac{(1-B)(1-A)}{2(A-B)} a x-\frac{1}{2}(c-x-1)-\frac{1+B}{2(1-B)} .
\end{aligned}
$$

Condition (2.1) shows that

$$
\begin{aligned}
p_{1} & =-\frac{1}{2}(c-x-1)+\frac{(1+B)(1+A) a x}{2(A-B)} \\
& <-\frac{1}{2}\left(c-1-\left|1+\frac{(1+B)(1+A) a}{A-B}\right|\right)<0 .
\end{aligned}
$$

Since $\max _{\rho \in \mathbb{R}}\left\{p_{1} \rho^{2}+q_{1} \rho+r_{1}\right\}=\left(4 p_{1} r_{1}-q_{1}^{2}\right) /\left(4 p_{1}\right)$ for $p_{1}<0$, it is clear that $Q(\rho)<0$ when

$$
\frac{(1-A B)^{2}}{(A-B)^{2}} a^{2} y^{2}<\left((c-x-1)-\frac{(1+B)(1+A)}{A-B} a x\right)
$$




$$
\times\left((c-x-1)+\frac{(1-B)(1-A)}{A-B} a x+\frac{1+B}{1-B}\right)
$$

$|x|,|y|<1$. As $y^{2}<1-x^{2}$, the above condition holds whenever

$$
\begin{aligned}
\frac{(1-A B)^{2}}{(A-B)^{2}} a^{2}\left(1-x^{2}\right) \leq((c & \left.-x-1)-\frac{(1+B)(1+A) a x}{A-B}\right) \\
& \times\left((c-x-1)+\frac{(1-B)(1-A)}{A-B} a x+\frac{1+B}{1-B}\right),
\end{aligned}
$$

that is, when

$$
\begin{gathered}
\left(a^{2}+2 a \frac{A+B}{A-B}+1\right) x^{2}-\left(2(c-1)\left(a \frac{A+B}{A-B}+1\right)+\right. \\
\left.\frac{1+B}{1-B}\left(1+a \frac{(1+A)(1+B)}{A-B}\right)\right) x+(c-1)^{2}+(c-1) \frac{1+B}{1-B}-a^{2} \frac{(1-A B)^{2}}{(A-B)^{2}} \geq 0 .
\end{gathered}
$$

To establish inequality (2.12), consider the polynomial $R$ given by

$$
R(x):=m x^{2}+n x+r, \quad|x|<1,
$$

where

$$
\begin{aligned}
m & :=a^{2}+2 a \frac{A+B}{A-B}+1=\frac{A(1+a)^{2}-B(1-a)^{2}}{A-B} \\
n & :=-2(c-1)\left(a \frac{A+B}{A-B}+1\right)-\frac{1+B}{1-B}\left(1+\frac{(1+A)(1+B)}{A-B} a\right), \\
r & :=(c-1)^{2}+(c-1) \frac{(1+B)}{1-B}-a^{2} \frac{(1-A B)^{2}}{(A-B)^{2}} .
\end{aligned}
$$

The constraint (2.3) yields $|n| \geq 2|m|$, and thus $R(x) \geq m+r-|n|$. Now inequality (2.2) readily implies that

$$
\begin{aligned}
R(x) \geq & m+r-|n| \\
= & a^{2}+2 a \frac{A+B}{A-B}+1+(c-1)^{2}+\frac{(c-1)(1+B)}{1-B}-\frac{a^{2}(1-A B)^{2}}{(A-B)^{2}} \\
& \quad-\left|2(c-1)\left(a \frac{A+B}{A-B}+1\right)+\frac{1+B}{1-B}\left(1+\frac{(1+A)(1+B)}{A-B} a\right)\right| \\
= & (c-1)^{2}-\left|2(c-1)\left(a \frac{A+B}{A-B}+1\right)+\frac{1+B}{1-B}\left(1+\frac{(1+A)(1+B)}{A-B} a\right)\right| \\
& +\frac{(c-1)(1+B)}{1-B}-\frac{\left(1-A^{2}\right)\left(1-B^{2}\right)}{(A-B)^{2}} a^{2}+2 \frac{A+B}{A-B} a+1 \geq 0 .
\end{aligned}
$$

Now considers the case of the constraint (2.5), which is equivalent to $|n|<2 m$. Then the minimum of $R$ occurs at $x=-n /(2 m)$, and (2.4) yields

$$
R(x) \geq \frac{4 m r-n^{2}}{4 m} \geq 0
$$


Evidently $\Psi$ satisfies the hypothesis of Lemma 1.1, and thus $\operatorname{Re} p(z)>0$, that is,

$$
-\frac{(1-A)-(1-B) \Phi(z)}{(1+A)-(1+B) \Phi(z)} \prec \frac{1+z}{1-z} .
$$

Hence there exists an analytic self-map $w$ of $\mathbb{D}$ with $w(0)=0$ such that

$$
-\frac{(1-A)-(1-B) \Phi(z)}{(1+A)-(1+B) \Phi(z)}=\frac{1+w(z)}{1-w(z)}
$$

which implies that $\Phi(z) \prec(1+A z) /(1+B z)$.

Theorem 2.1 gives rise to simple conditions on $a$ and $c$ to ensure $\Phi(a ; c ; z)$ maps $\mathbb{D}$ into a half-plane.

Corollary 2.1. Let $a \leq 0$ and $c \geq 2\left(1+a^{2}\right)$. Then $\operatorname{Re} \Phi(a ; c ; z)>a /(a-1)$.

Proof. The result follows from Theorem 2.1 by choosing $A=-(a+1) /(a-1)$, and $B=-1$. In this case, condition (2.1) reduces to $c \geq 2$ which clearly holds for $c \geq 2\left(1+a^{2}\right)$. Also (2.3) and (2.2) respectively reduces to

$$
\left(1+a^{2}\right) c-2\left(1+2 a^{2}\right) \geq 0
$$

and

$$
(c-1)^{2}-2(c-1)\left(a^{2}+1\right) \geq-2 a^{2}-1 .
$$

Since $c \geq 2+2 a^{2}$,

$$
\left(1+a^{2}\right) c-2\left(1+2 a^{2}\right) \geq 2\left(1+a^{2}\right)^{2}-2\left(1+2 a^{2}\right)=2 a^{4} \geq 0,
$$

and

$$
(c-1)^{2}-2(c-1)\left(a^{2}+1\right)+2 a^{2}+1=(c-2)\left(c-2-2 a^{2}\right) \geq 0,
$$

which establishes both (2.13) and (2.14).

Remark 2.1. It is noteworthy to compare the result obtained in Corollary 2.1 with a result of Ponnusamy and Vuorinen [10]. With $\beta:=a /(a-1), a \in(-1,0]$, the result of Ponnusamy and Vuorinen [10, Theorem 1.9, p.77] gives $\operatorname{Re} \Phi(a ; c ; z)>a /(a-1)$ provided $c \geq 1+\sqrt{\left(1+3 a^{2}\right) /\left(1-a^{2}\right)}$. Since $1+\sqrt{\left(1+3 a^{2}\right) /\left(1-a^{2}\right)} \geq 2+2 a^{2}$, it is clear that Corollary 2.1 extends the range of $c$. Further, since $a \leq 0$, Corollary 2.1 extends the range of $\beta$ to the whole interval $\beta \in[0,1)$.

Corollary 2.2. Let $a>0$ and

$$
c \geq \begin{cases}2, & a \in(0,1) \\ 1+a^{2}, & a \geq 1 .\end{cases}
$$

Then

$$
\operatorname{Re} \Phi(a ; c ; z)>\frac{1+a^{2}}{(1+a)^{2}} \geq \frac{1}{2}
$$


Proof. Let $A=-(1-a)^{2} /(1+a)^{2}$, and $B=-1$ in Theorem 2.1. In this case, (2.1) reduces to $c \geq 2$, which clearly holds. Now condition (2.3) is trivially true. Thus the result follows from Theorem 2.1 provided (2.2) holds, or equivalently, if

$$
(c-1)^{2}-(c-1)\left|1-a^{2}\right| \geq a^{2} .
$$

Let $a \in(0,1)$ and $c \geq 2$. Then

$$
(c-1)^{2}-(c-1)\left|1-a^{2}\right|=(c-1)(c-2)+(c-1) a^{2} \geq a^{2} .
$$

If $a \geq 1, c \geq 1+a^{2}$, then

$$
(c-1)^{2}-(c-1)\left|1-a^{2}\right|=(c-1)\left(c-a^{2}\right) \geq a^{2} .
$$

Thus (2.15) holds in either case. From Theorem 2.1, it is evident that $\Phi(a ; c ; z) \in$ $\mathcal{P}[A,-1]$, or equivalently,

$$
\operatorname{Re} \Phi(a ; c ; z)>\frac{1-A}{2}=\frac{1+a^{2}}{(1+a)^{2}} .
$$

Remark 2.2. Corollary 2.2 improves a result in [10, Corollary 1.11, p.77] to the wider range $a \in\left(0, a_{0}\right]$, where $a_{0}=1.27202$ is a root of $a^{4}-a^{2}-1=0$. It also improves [10, Corollary 1.12, p.78] for $a \in(0,1)$.

Corollary 2.3. Let $a, c$ be real such that

$$
c \geq \begin{cases}2-2 a, & a \leq 0 \\ 2, & 0 \leq a \leq 1 \\ 2 a, & a \geq 1\end{cases}
$$

Then $\operatorname{Re} \Phi(a ; c ; z)>1 / 2$.

Proof. Put $A=0$ and $B=-1$ in Theorem 2.1. The condition (2.1) reduces to $c \geq 2$, which holds in all cases. It is sufficient to establish conditions (2.3) and (2.2), or equivalently,

$$
c-1-|1-a| \geq 0
$$

and

$$
(c-1)^{2}-2(c-1)|1-a|-2 a+1 \geq 0 .
$$

Consider the case when $a \leq 0$ and $c \geq 2-2 a$. Then $c-1-|1-a|=c-2+$ $a \geq-a \geq 0$, and $(c-1)^{2}-2(c-1)|1-a|-2 a+1=(c-2)(c-2+2 a) \geq 0$. Thus both (2.16) and (2.17) hold.

For $a \in[0,1]$ and $c \geq 2$, then $c-1-|1-a|=c-2+a \geq a \geq 0$, and $(c-1)^{2}-2(c-1)|1-a|-2 a+1=(c-2)(c-2+2 a) \geq 0$.

Finally it is readily established for $a \geq 1$ and $c \geq 2 a$ that $c-1-|1-a|=$ $c-a \geq a \geq 0$, and $(c-1)^{2}-2(c-1)|1-a|-2 a+1=c(c-2 a) \geq 0$.

Remark 2.3. Corollary 2.3 encompasses (for $a \geq 1$ ) a result in [10, Theorem 1.13, p.78]. It also expands the range of a to include all real numbers. 
Corollary 2.4. Let $a \geq-1 / 2, a \neq 0$, and

$$
c \geq \begin{cases}2, & a \in[-1 / 2,0) \\ 2 a+3, & a>0\end{cases}
$$

Then $(1+a)|\Phi(a ; c ; z)-1|<|a|$. In particular,

$$
\left|e^{z / 2}\left((3-2 z) I_{0}\left(\frac{z}{2}\right)+(2 z-1) I_{1}\left(\frac{z}{2}\right)\right)-3\right|<3
$$

where $I_{n}(z)$ is the modified Bessel function of order $n$.

Proof. Choose $A=|a| /(a+1)$ and $B=0$ in Theorem 2.1. First consider the case when $a>0$. The condition (2.1) reduces to $c \geq 2 a+3$, and condition (2.2), which is equivalent to $(c-1)(c-2 a-3) \geq 0$, clearly holds. Now (2.3) reduces to $c \geq 1+a$, which holds because $c \geq 2 a+3, a>0$. From Theorem 2.1 it is evident that $\Phi(a ; c ; z) \prec 1+a z /(1+a)$, that is, $|\Phi(a ; c ; z)-1|<a /(1+a)$.

Now consider the case when $a \in[-1 / 2,0)$. Then (2.1) gives $c \geq 2$. Conditions (2.2) and (2.3) respectively become

$$
c(c-1)-2|(c-1)(1+a)|>0,
$$

and

$$
|-2 a(c-1)|+2 a(1+a)>0 .
$$

Since $c \geq 2$ and $a \in[-1 / 2,0)$,

$c(c-1)-2|(c-1)(1+a)|=c(c-1)-2(c-1)(1+a)=(c-1)(c-2-2 a)>0$,

and

$$
|-2 a(c-1)|+2 a(1+a)=-2 a(c-1)+2 a+2 a^{2}=-2 a(c-2)+2 a^{2}>0,
$$

which established both (2.18) and (2.19). Again from Theorem 2.1 it follows that

$$
(1+a)|\Phi(a ; c ; z)-1|<-a .
$$

An application of the identity

$$
\Phi\left(-\frac{1}{2} ; 2 ; z\right)=\frac{e^{z / 2}}{3}\left((3-2 z) I_{0}\left(\frac{z}{2}\right)+(2 z-1) I_{1}\left(\frac{z}{2}\right)\right)
$$

establishes the final assertion.

Theorem 2.2. Let $3-2 \sqrt{2}<B<A \leq 1$, and $a, c \in \mathbb{R}$ satisfy

$$
c-1 \geq \max \left\{1,\left|1+\frac{(1+B)(1+A)}{A-B} a\right|\right\} \text {. }
$$

Suppose $A, B, c$ and a satisfy

$$
(c-1)^{2}-2\left|(c-1)\left(\frac{A+B}{A-B} a+1\right)+\frac{8 B(1-B)}{(1+B)^{3}}\left(1+\frac{(1+A)(1+B)}{A-B} a\right)\right|
$$




$$
+16(c-1) \frac{B(1-B)}{(1+B)^{3}} \geq \frac{\left(1-A^{2}\right)\left(1-B^{2}\right)}{(A-B)^{2}} a^{2}-2 \frac{A+B}{A-B} a-1
$$

whenever

$$
\begin{gathered}
\left|(c-1)\left(\frac{A+B}{A-B} a+1\right)+\frac{8 B(1-B)}{(1+B)^{3}}\left(1+\frac{(1+A)(1+B)}{A-B} a\right)\right| \\
\geq\left|\frac{A(1+a)^{2}-B(1-a)^{2}}{A-B}\right|
\end{gathered}
$$

or the inequality

$$
\begin{aligned}
& \left((c-1)\left(\frac{a(A+B)}{(A-B)}+1\right)+\frac{8 B(1-B)}{(1+B)^{3}}\left(1+\frac{(1+A)(1+B)}{A-B} a\right)\right)^{2} \\
& \leq\left(1+2 a \frac{A+B}{A-B}+a^{2}\right)\left((c-1)^{2}+(c-1) \frac{16 B(1-B)}{(1+B)^{3}}-a^{2} \frac{(1-A B)^{2}}{(A-B)^{2}}\right)
\end{aligned}
$$

when

$$
\begin{aligned}
& \left|(c-1)\left(\frac{A+B}{A-B} a+1\right)+\frac{8 B(1-B)}{(1+B)^{3}}\left(1+\frac{(1+A)(1+B)}{A-B} a\right)\right| \\
& <\frac{A(1+a)^{2}-B(1-a)^{2}}{A-B} . \\
& \text { If }(1+B) \Phi(a ; c ; z) \neq(1+A), \text { then } \Phi(a ; c ; z) \in \mathcal{P}[A, B] .
\end{aligned}
$$

Proof. Proceeding similarly as in the proof of Theorem 2.1, consider $\operatorname{Re} \Psi(i \rho, \sigma$, $\mu+i v ; z)$ as given in (2.11). For $\sigma \leq-\left(1+\rho^{2}\right) / 2, \rho \in \mathbb{R}$, and $B \geq 3-2 \sqrt{2}$,

$$
\frac{2\left(1-B^{2}\right)}{(1-B)^{2}+(1+B)^{2} \rho^{2}} \sigma^{2} \geq \frac{2\left(1-B^{2}\right)}{(1-B)^{2}+(1+B)^{2} \rho^{2}} \frac{\left(1+\rho^{2}\right)^{2}}{4} \geq \frac{8 B(1-B)}{(1+B)^{3}} .
$$

With $z=x+i y \in \mathbb{D}$, and $\mu+\sigma<0$, it follows that

$$
\begin{aligned}
\operatorname{Re} \Psi(i \rho, \sigma, \mu+i v ; z) \leq- & \frac{1}{2}(c-x-1)\left(1+\rho^{2}\right)+\frac{(1+B)(1+A) \rho^{2}}{2(A-B)} a x \\
& \quad+\frac{\rho(1-A B)}{(A-B)} a y-\frac{(1-B)(1-A)}{2(A-B)} a x-\frac{8 B(1-B)}{(1+B)^{3}} \\
= & p_{2} \rho^{2}+q_{2} \rho+r_{2}:=Q_{1}(\rho),
\end{aligned}
$$

where

$$
\begin{aligned}
& p_{2}=-\frac{1}{2}(c-x-1)+\frac{(1+B)(1+A) a x}{2(A-B)}, \\
& q_{2}=\frac{(1-A B) a y}{(A-B)} \\
& r_{2}=-\frac{(1-B)(1-A)}{2(A-B)} a x-\frac{1}{2}(c-x-1)-\frac{8 B(1-B)}{(1+B)^{3}} .
\end{aligned}
$$


As per the proof of Theorem 2.1, observe that the constraint (2.20) implies that $p_{2}<0$. Thus $Q_{1}(\rho)<0$ for all $\rho \in \mathbb{R}$ provided $q_{2}^{2} \leq 4 p_{2} r_{2}$, that is,

$$
\begin{aligned}
\frac{(1-A B)^{2}}{(A-B)^{2}} a^{2} y^{2} \leq((c-x-1) & \left.-\frac{(1+B)(1+A)}{(A-B)} a x\right) \\
\times & \left((c-x-1)+\frac{(1-B)(1-A)}{(A-B)} a x+\frac{16 B(1-B)}{(1+B)^{3}}\right)
\end{aligned}
$$

$|x|,|y|<1$. With $y^{2}<1-x^{2}$, it is sufficient to show

$$
\begin{aligned}
\frac{(1-A B)^{2}}{(A-B)^{2}} a^{2}\left(1-x^{2}\right) \leq( & \left.(c-x-1)-\frac{(1+B)(1+A) a x}{(A-B)}\right) \\
& \times\left((c-x-1)+\frac{(1-B)(1-A)}{(A-B)} a x+\frac{16 B(1-B)}{(1+B)^{3}}\right)
\end{aligned}
$$

for $|x|<1$. The above inequality is equivalent to showing

$$
R_{1}(x):=m_{1} x^{2}+n_{1} x+r_{1} \geq 0,
$$

where

$$
\begin{aligned}
m_{1} & :=1+2 a \frac{A+B}{A-B}+a^{2} \\
n_{1} & :=-2\left((c-1)\left(\frac{a(A+B)}{(A-B)}+1\right)+\frac{8 B(1-B)}{(1+B)^{3}}\left(1+\frac{(1+A)(1+B)}{A-B} a\right)\right), \\
r_{1} & :=(c-1)^{2}+(c-1) \frac{16 B(1-B)}{(1+B)^{3}}-\frac{a^{2}(1-A B)^{2}}{(A-B)^{2}} .
\end{aligned}
$$

If (2.22) holds, then $\left|n_{1}\right| \geq 2\left|m_{1}\right|$. Since $R_{1}$ is increasing, then $R_{1}(x) \geq m_{1}+$ $r_{1}-\left|n_{1}\right|$, which is nonnegative from (2.21). On the other hand, if (2.24) holds, then $\left|n_{1}\right|<2\left|m_{1}\right|, R_{1}(x) \geq\left(4 m_{1} r_{1}-n_{1}^{2}\right) / 4 m_{1}$, and (2.23) implies $R_{1}(x) \geq 0$. Either case establishes (2.25).

The following results are immediate consequences from relation (1.3) and respectively Theorem 2.1 and Theorem 2.2.

Theorem 2.3. Let $-1 \leq B \leq 3-2 \sqrt{2} \approx 0.171573$. Suppose $B<A \leq 1, a, c \in \mathbb{R}$ with $a \neq 0$ and satisfying

$$
c \geq \max \left\{1,\left|1+\frac{(1+B)(1+A)}{A-B}(a+1)\right|\right\} .
$$

Further let $A, B, c$ and a satisfy either

$$
\begin{gathered}
c^{2}-\left|2 c\left((a+1) \frac{A+B}{A-B}+1\right)+\frac{1+B}{1-B}\left(1+\frac{(1+A)(1+B)}{A-B}(a+1)\right)\right| \\
+\frac{c(1+B)}{1-B} \geq \frac{\left(1-A^{2}\right)\left(1-B^{2}\right)}{(A-B)^{2}}(a+1)^{2}-2 \frac{A+B}{A-B}(a+1)-1
\end{gathered}
$$


whenever

$$
\begin{gathered}
|2 c(1-B)((a+1)(A+B)+A-B)+(1+B)(A-B+(1+A)(1+B)(a+1))| \\
\geq 2(1-B)\left|A(2+a)^{2}-B a^{2}\right|
\end{gathered}
$$

or the inequality

$$
\begin{aligned}
& \left(2 c \frac{A(2+a)+B a}{A-B}+\frac{1+B}{1-B}\left(1+\frac{(1+A)(1+B)}{A-B}(a+1)\right)\right)^{2} \\
& \leq 4\left((a+1)^{2}+\frac{2(A+B)}{A-B}(a+1)+1\right)\left(c^{2}+\frac{1+B}{1-B} c-\frac{(1-A B)^{2}}{(A-B)^{2}}(a+1)^{2}\right)
\end{aligned}
$$

when

$$
\begin{gathered}
|2 c(1-B)((a+1)(A+B)+A-B)+(1+B)(A-B+(1+A)(1+B)(a+1))| \\
<2(1-B)\left(A(2+a)^{2}-B a^{2}\right) . \\
\text { If }(1+B) \Phi(a+1 ; c+1 ; z) \neq(1+A), \text { then }(c / a) \Phi^{\prime}(a ; c ; z) \in \mathcal{P}[A, B] .
\end{gathered}
$$

Theorem 2.4. Let $3-2 \sqrt{2}<B<A \leq 1$. Suppose $a, c \in \mathbb{R}, a \neq 0$, such that

$$
c \geq \max \left\{1,\left|1+\frac{(1+B)(1+A)}{A-B}(1+a)\right|\right\} \text {. }
$$

Further let $A, B, c$ and a satisfy

$$
\begin{gathered}
c^{2}-2\left|c\left(\frac{A+B}{A-B}(a+1)+1\right)+\frac{8 B(1-B)}{(1+B)^{3}}\left(1+\frac{(1+A)(1+B)}{A-B}(a+1)\right)\right| \\
+16 c \frac{B(1-B)}{(1+B)^{3}} \geq \frac{\left(1-A^{2}\right)\left(1-B^{2}\right)}{(A-B)^{2}}(a+1)^{2}-2 \frac{A+B}{A-B}(a+1)-1
\end{gathered}
$$

when

$$
\begin{gathered}
\left|c\left(\frac{A+B}{A-B}(a+1)+1\right)+\frac{8 B(1-B)}{(1+B)^{3}}\left(1+\frac{(1+A)(1+B)}{A-B}(a+1)\right)\right| \\
\geq\left|\frac{A(2+a)^{2}-B a^{2}}{A-B}\right|
\end{gathered}
$$

or the inequality

$$
\begin{aligned}
& \left(c\left(\frac{A+B}{A-B}(a+1)+1\right)+\frac{8 B(1-B)}{(1+B)^{3}}\left(1+\frac{(1+A)(1+B)}{A-B}(a+1)\right)^{2}\right. \\
& \quad \leq\left(1+2(a+1) \frac{A+B}{A-B}+a^{2}\right)\left(c^{2}+c \frac{16 B(1-B)}{(1+B)^{3}}-\frac{(a+1)^{2}(1-A B)^{2}}{(A-B)^{2}}\right)
\end{aligned}
$$

when

$$
\begin{aligned}
& \left|c\left(\frac{A+B}{A-B}(a+1)+1\right)+\frac{8 B(1-B)}{(1+B)^{3}}\left(1+\frac{(1+A)(1+B)}{A-B}(a+1)\right)\right| \\
& <\frac{A(2+a)^{2}-B a^{2}}{A-B} \text {. } \\
& \text { If }(1+B) \Phi(a+1 ; c+1 ; z) \neq(1+A) \text {, then }(c / a) \Phi^{\prime}(a ; c ; z) \in \mathcal{P}[A, B] \text {. }
\end{aligned}
$$


With $B=-1$, the following results are easily deduced from Theorem 2.3 by choosing respectively $A=-(a+2) / a, A=-a^{2} /(2+a)^{2}$ and $A=0$.

Corollary 2.5. Let $a \leq-1$, and $c \geq 1+2(1+a)^{2}$. Then $(c / a)(\Phi(a ; c ; z)-1)$ is close-to-convex of order $(a+1) / a$ with respect to the identity function.

Corollary 2.6. Let $a>-1, a \neq 0$, and

$$
c \geq \begin{cases}1, & a \in(-1,0) \\ a^{2}, & a>0\end{cases}
$$

Then $(c / a)(\Phi(a ; c ; z)-1)$ is close-to-convex of order $\left(2+2 a+a^{2}\right) /(2+a)^{2}$ with respect to the identity function.

Corollary 2.7. Let a be a nonzero real number, and

$$
c \geq \begin{cases}-1-2 a, & a \leq-1 \\ 1, & -1 \leq a<0 \\ 1+2 a, & a>0\end{cases}
$$

Then $\operatorname{Re}(c / a) \Phi^{\prime}(a ; c ; z)>1 / 2$.

\section{Examples}

The associated Laguerre polynomial $L_{n}^{\alpha}[4]$ is given by the series

$$
L_{n}^{\alpha}(z):=\sum_{k=0}^{n}(-1)^{k} \frac{\Gamma(n+\alpha+1)}{(n-k) ! \Gamma(\alpha+k+1) k !} z^{k} .
$$

It relates to the confluent hypergeometric function via the identity

$$
(\alpha+1)_{n} \Phi(-n ; \alpha+1 ; z)=n ! L_{n}^{\alpha}(z)
$$

Thus

$$
\frac{m !}{\left(1+\frac{m^{4}}{4}\right)_{m}} L_{m}^{\frac{m^{4}}{4}}(z)=\Phi\left(-m ; 1+\frac{m^{4}}{4} ; z\right) .
$$

The associated Laguerre polynomial $L_{n}^{\alpha}$ is used to illustrate the significance of Theorem 2.1.

Example 3.1. For every $m=2,3, \ldots$,

$$
\frac{m !}{\left(1+\frac{m^{4}}{4}\right)_{m}} L_{m}^{\frac{m^{4}}{4}}(z) \prec \frac{1+\frac{z}{(1-m)^{2}}}{1+\frac{z}{(1+m)^{2}}},
$$

provided $(1+m)^{2}\left(2-2 m+m^{2}\right)\left(1+m^{4} / 4\right)_{m} \neq m !(1-m)^{2}\left(2+2 m+m^{2}\right) L_{m}^{m^{4} / 4}(z)$. 
To verify the subordination given in (3.1), consider $p: \mathbb{D} \rightarrow \mathbb{C}$ given by

$$
p(z):=-\frac{\left(1-\frac{1}{(1-m)^{2}}\right)-\left(1-\frac{1}{(1+m)^{2}}\right) \Phi_{1}(z)}{\left(1+\frac{1}{(1-m)^{2}}\right)-\left(1+\frac{1}{(1+m)^{2}}\right) \Phi_{1}(z)}
$$

where $\Phi_{1}(z):=\Phi\left(-m ; 1+m^{4} / 4 ; z\right)$. Proceeding as in the proof of the Theorem 2.1 with $A=1 /(1-m)^{2}$ and $B=1 /(1+m)^{2}$, then (2.11) reduces to

$$
\begin{aligned}
\operatorname{Re} \Psi(i \rho, \sigma, \mu+i v ; z)=\mu & -\frac{2 m(m+2)\left(m^{2}+2 m+2\right)}{m^{2}(m+2)^{2}+\left(m^{2}+2 m+2\right)^{2} \rho^{2}} \sigma^{2}+\left(1+\frac{m^{4}}{4}-x\right) \sigma \\
& -\frac{\rho}{4} m^{2}\left(m^{2}-2\right) y+\frac{1}{8} m^{2}\left(m^{2}-4\right) x-\frac{1}{8}\left(m^{4}+4\right) \rho^{2} x
\end{aligned}
$$

Now $\sigma \leq-\left(1+\rho^{2}\right) / 2$ yields

$$
\begin{aligned}
& \frac{2 m(m+2)\left(m^{2}+2 m+2\right)}{m^{2}(m+2)^{2}+\left(m^{2}+2 m+2\right)^{2} \rho^{2}} \sigma^{2} \geq \\
& \frac{2 m(m+2)\left(m^{2}+2 m+2\right)}{m^{2}(m+2)^{2}+\left(m^{2}+2 m+2\right)^{2} \rho^{2}} \frac{\left(1+\rho^{2}\right)^{2}}{4}:=h(\rho) .
\end{aligned}
$$

Taking logarithmic derivatives, it is evident that $h$ is increasing for $\rho \geq 0$, and thus $h(\rho) \geq\left(m^{2}+2 m+2\right) / 2 m(m+2)$. Now as $\mu+\sigma<0$, (3.2) yields

$$
\begin{aligned}
\operatorname{Re} \Psi(i \rho, \sigma, \mu+i v ; z) \leq & (\mu+\sigma)-\frac{m^{2}+2 m+2}{2 m(m+2)}-\frac{1}{2}\left(1+\frac{m^{4}}{4}-x-1\right)\left(1+\rho^{2}\right) \\
& \quad-\frac{\rho}{4} m^{2}\left(m^{2}-2\right) y+\frac{1}{8} m^{2}\left(m^{2}-4\right) x-\frac{1}{8}\left(m^{4}+4\right) \rho^{2} x \\
& <p_{1} \rho^{2}+q_{1} \rho+r_{1},
\end{aligned}
$$

where

$$
\begin{aligned}
& p_{1}=-\frac{m^{4}}{8}(1+x)<0, \\
& q_{1}=-\frac{1}{4} m^{2}\left(m^{2}-2\right) y, \\
& r_{1}=-\frac{m^{2}+2 m+2}{2 m(m+2)}-\frac{m^{4}}{8}+\frac{1}{8}\left(m^{2}-2\right)^{2} x .
\end{aligned}
$$

As $|x|<1,|y|<1$ and $y^{2}<1-x^{2}$, it follows that

$$
\begin{aligned}
q_{1}^{2}-4 p_{1} r_{1}= & \frac{1}{16} m^{4}\left(m^{2}-2\right)^{2} y^{2} \\
& \quad+\frac{m^{4}}{2}(1+x)\left(\frac{1}{8}\left(m^{2}-2\right)^{2} x-\frac{m^{4}}{8}-\frac{m^{2}+2 m+2}{2 m(m+2)}\right) \\
= & \frac{1}{16} m^{4}\left(m^{2}-2\right)^{2}\left(x^{2}+y^{2}\right)+\frac{1}{16} m^{4}\left(m^{2}-2\right)^{2} x
\end{aligned}
$$




$$
\begin{aligned}
& -\frac{m^{4}}{2}\left(\frac{m^{4}}{8}+\frac{m^{2}+2 m+2}{2 m(m+2)}\right)(1+x) \\
<- & \left(\frac{m^{6}}{4}+\frac{m^{3}}{2(m+2)}\right)(1+x)<0 .
\end{aligned}
$$

Since $p_{1}<0$, the above inequality implies that $\max \left(p_{1} \rho^{2}+q_{1} \rho+r_{1}\right)=$ $\left(4 p_{1} r_{1}-q_{1}^{2}\right) /\left(4 p_{1}\right)<0$ over $\rho \in \mathbb{R}$. Hence $\operatorname{Re} \Psi(i \rho, \sigma, \mu+i v ; z)<0$. The final assertion now follows readily by using the same arguments as in the proof of Theorem 2.1.

The next example illustrates both Theorem 2.1 and Theorem 2.2 in the sense that the real parameters satisfy the conditions of each theorem within different constraint intervals.

Example 3.2. If $a \in(-\infty,-1)$ and $c \geq \max \left\{2,1+a^{4} / 4\right\}$, then

$$
\Phi(a ; c ; z) \prec \frac{1+\frac{z}{(1+a)^{2}}}{1+\frac{z}{(1-a)^{2}}}, \quad z \in \mathbb{D},
$$

provided $(1-a)^{2}\left(2+2 a+a^{2}\right) \neq(1+a)^{2}\left(a^{2}-2 a\right) \Phi(a ; c ; z)$.

To establish the subordination (3.3), define the analytic function $p: \mathbb{D} \rightarrow \mathbb{C}$ by

$$
p(z):=-\frac{\left(1-\frac{1}{(1+a)^{2}}\right)-\left(1-\frac{1}{(1-a)^{2}}\right) \Phi(a ; c ; z)}{\left(1+\frac{1}{(1+a)^{2}}\right)-\left(1+\frac{1}{(1-a)^{2}}\right) \Phi(a ; c ; z)}
$$

Proceeding similarly as in the proof of the Theorem 2.1 with $A=1 /(1+a)^{2}$ and $B=1 /(1-a)^{2}$, the equivalent form for $(2.11)$ is

$$
\begin{aligned}
\operatorname{Re} \Psi(i \rho, \sigma, \mu+i v ; z)=\mu & -\frac{2 a(a-2)\left(a^{2}-2 a+2\right)}{(a-2)^{2} a^{2}+((a-2) a+2)^{2} \rho^{2}} \sigma^{2}+(c-x) \sigma \\
& -\frac{\rho}{4} a^{2}\left(a^{2}-2\right) y+\frac{1}{8} a^{2}\left(a^{2}-4\right) x-\frac{1}{8}\left(a^{4}+4\right) \rho^{2} x
\end{aligned}
$$

Now $\sigma \leq-\left(1+\rho^{2}\right) / 2$ yields

$$
\begin{aligned}
\frac{2\left((a-1)^{4}-1\right)}{(a-2)^{2} a^{2}+((a-2) a+2)^{2} \rho^{2}} \sigma^{2} & \geq \frac{2\left((a-1)^{4}-1\right)}{(a-2)^{2} a^{2}+((a-2) a+2)^{2} \rho^{2}} \frac{\left(1+\rho^{2}\right)^{2}}{4} \\
& :=\frac{a(a-2)\left(a^{2}-2 a+2\right)}{2} h(\rho),
\end{aligned}
$$

where $h(\rho)=\left(1+\rho^{2}\right)^{2} /\left((a-2)^{2} a^{2}+((a-2) a+2)^{2} \rho^{2}\right)>0$. By taking logarithmic derivatives, it follows that

$$
h^{\prime}(\rho)=\frac{\left(a^{4}-4 a^{3}+8 a-4\right)+((a-2) a+2)^{2} \rho^{2}}{\left(1+\rho^{2}\right)\left((a-2)^{2} a^{2}+((a-2) a+2)^{2} \rho^{2}\right)} \rho h(\rho) .
$$


We next consider two cases. First is when $a \in(-\infty,-\sqrt{2}]$, that is, $B \leq 3-2 \sqrt{2}$. In this case, it follows from (3.5) that $h$ is increasing for $\rho \geq 0$, and hence $h(\rho) \geq$ $1 /\left((a-2)^{2} a^{2}\right)$. Thus

$$
\begin{aligned}
\operatorname{Re} \Psi(i \rho, \sigma, \mu+i v ; z) \leq & (\mu+\sigma)-\frac{a^{2}-2 a+2}{2 a(a-2)}+(c-x-1) \sigma \\
& \quad-\frac{\rho}{4} a^{2}\left(a^{2}-2\right) y+\frac{1}{8} a^{2}\left(a^{2}-4\right) x-\frac{1}{8}\left(a^{4}+4\right) \rho^{2} x \\
= & p_{1} \rho^{2}+q_{1} \rho+r_{1},
\end{aligned}
$$

where

$$
\begin{aligned}
p_{1} & =-\frac{1}{2}(c-1)-\frac{a^{4}}{8} x<-\frac{1}{2}(c-1)+\frac{a^{4}}{8} \leq 0 \\
q_{1} & =-\frac{1}{4} a^{2}\left(a^{2}-2\right) y \\
r_{1} & =-\frac{a^{2}-2 a+2}{2(a-2) a}-\frac{1}{2}(c-x-1)+ \\
& =-\frac{1}{8} a^{2}\left(a^{2}-4\right) x \\
& =-\frac{1}{a(a-2)}-\frac{c}{2}+\frac{1}{8}\left(a^{2}-2\right)^{2} x
\end{aligned}
$$

Since $|x|<1,|y|<1$ and $y^{2}<1-x^{2}$, a computation gives

$$
\begin{aligned}
q_{1}^{2}-4 p_{1} r_{1}= & \frac{1}{16} a^{4}\left(a^{2}-2\right)^{2} y^{2}-\left(c-1+\frac{a^{4}}{4} x\right)\left(\frac{2}{a(a-2)}+c-\frac{1}{4}\left(a^{2}-2\right)^{2} x\right) \\
< & \frac{1}{16} a^{4}\left(a^{2}-2\right)^{2}-\frac{1}{4}(c-1)\left(a^{2}-2\right)^{2} x+\frac{a^{4}}{4}\left(\frac{1}{a(a-2)}+c\right) x \\
& \quad-(c-1)\left(\frac{1}{a(a-2)}+c\right) \\
< & \frac{1}{16} a^{4}\left(a^{2}-2\right)^{2}-\frac{1}{4}(c-1)\left(a^{2}-2\right)^{2}+\frac{a^{4}}{4}\left(\frac{1}{a(a-2)}+c\right) \\
& \quad-(c-1)\left(\frac{1}{a(a-2)}+c\right) \\
= & \frac{1}{16}\left(\left(a^{2}-2\right)^{2}+\frac{4}{a(a-2)}+c\right)\left(a^{4}-4(c-1)\right) \leq 0 .
\end{aligned}
$$

Since $\max \left(p_{1} \rho^{2}+q_{1} \rho+r_{1}\right)=\left(4 p_{1} r_{1}-q_{1}^{2}\right) /\left(4 p_{1}\right)<0$ for $\rho \in \mathbb{R}$, it follows that $\operatorname{Re} \Psi(i \rho, \sigma, \mu+i v ; z)<0$. The final assertion for the case $a \in(-\infty,-\sqrt{2}]$ follows by adopting the same arguments used in the proof of Theorem 2.1.

Next consider the case when $a \in(-\sqrt{2},-1)$, that is, the case $B \geq 3-2 \sqrt{2}$. For this range of $a$, the expression $a^{4}-4 a^{3}+8 a-4$ is negative, and hence $h^{\prime}$ given in (3.5) is non-negative when $\rho>0$ and $\rho^{2} \geq-\left(a^{4}-4 a^{3}+8 a-4\right) /\left(a^{2}-2 a+2\right)^{2}$. Together these imply that

$$
h(\rho) \geq \frac{16(a-1)^{2}}{\left(a^{2}-2 a+2\right)^{4}}
$$


It is immediate from (3.4) that

$$
\begin{aligned}
\operatorname{Re} \Psi(i \rho, \sigma, \mu+i v ; z) \leq & (\mu+\sigma)-\frac{8 a(a-2)(a-1)^{2}}{\left(a^{2}-2 a+2\right)^{3}}+(c-x-1) \sigma \\
& \quad-\frac{\rho}{4} a^{2}\left(a^{2}-2\right) y+\frac{1}{8} a^{2}\left(a^{2}-4\right) x-\frac{1}{8}\left(a^{4}+4\right) \rho^{2} x \\
= & p_{2} \rho^{2}+q_{2} \rho+r_{2},
\end{aligned}
$$

where

$$
\begin{aligned}
& p_{2}=-\frac{1}{2}(c-1)-\frac{a^{4}}{8} x<-\frac{1}{2}(c-1)+\frac{a^{4}}{8}<0, \\
& q_{2}=-\frac{1}{4} a^{2}\left(a^{2}-2\right) y, \\
& r_{2}=-\frac{8 a(a-2)(a-1)^{2}}{\left(a^{2}-2 a+2\right)^{3}}-\frac{c-1}{2}+\frac{1}{8}\left(a^{2}-2\right)^{2} x .
\end{aligned}
$$

We need to show that $q_{2}^{2}-4 p_{2} r_{2}<0$. Since $|x|<1,|y|<1$ and $y^{2}<1-x^{2}$, a computation yields

$$
\begin{aligned}
q_{2}^{2}-4 p_{2} r_{2}= & \frac{a^{4}}{16}\left(a^{2}-2\right)^{2}\left(x^{2}+y^{2}\right)-(c-1)^{2}-\frac{16 a(a-2)(a-1)^{2}(c-1)}{\left(a^{2}-2 a+2\right)^{3}} \\
& -\left(\frac{a^{4}}{4}(c-1)+\frac{4 a^{5}(a-2)(a-1)^{2}}{\left(a^{2}-2 a+2\right)^{3}}-\frac{(c-1)\left(a^{2}-2\right)^{2}}{4}\right) x \\
< & \left(a^{4}-4(c-1)\right)\left(\frac{\left(a^{2}-2\right)^{2}}{16}+\frac{c-1}{4}+\frac{4 a(a-2)(a-1)^{2}}{\left(a^{2}-2 a+2\right)^{3}}\right) .
\end{aligned}
$$

Evidently, the conclusion follows if the expression

$$
M_{a}=\left(a^{2}-2 a+2\right)^{3}\left(a^{4}-2 a^{2}+2\right)+32 a(a-2)(a-1)^{2}
$$

is positive, which clearly holds for $a \in(-\sqrt{2},-1)$.

\section{Janowski starlikeness of the confluent hypergeometric func- tion}

This section looks at finding conditions to ensure a normalized confluent hypergeometric function $z \Phi(a ; c ; z)$ is Janowski starlike. For this purpose, we first derive sufficient conditions for $\Phi(a ; c ; z)$ to be Janowski convex, after which an application of relation (1.3) yields conditions for $z \Phi(a ; c ; z) \in \mathcal{S}^{*}[A, B]$.

Theorem 4.1. Let $a, c \in \mathbb{R}$ be such that $(A-B) \Phi^{\prime}(a ; c, z) \neq(1+B) z \Phi^{\prime \prime}(a ; c ; z)$, $-1 \leq B<A \leq 1$. Suppose

$$
c(1+B) \geq(1+B)\left|1+\frac{(1+a)(1+B)}{A-B}\right|-(A-B+1) .
$$


Further let $A, B, c$ and a satisfy either

$$
\begin{aligned}
& \left(1-B(A-B)+\left(2 B-(A-B)\left(1+B^{2}\right)\right) \frac{1+a}{A-B}+c\left(1-B^{2}\right)\left(1+\frac{B(1+a)}{A-B}\right)\right)^{2} \\
& \leq(A-B+1+c(1+B))(1-A+B+c(1-B))-\left(\frac{(1+a)\left(1-B^{2}\right)}{A-B}-B\right)^{2}
\end{aligned}
$$

whenever

$$
\left|1-B(A-B)+\left(2 B-(A-B)\left(1+B^{2}\right)\right) \frac{1+a}{A-B}+c\left(1-B^{2}\right)\left(1+\frac{B(1+a)}{A-B}\right)\right| \leq 1,
$$

or the inequality

$$
\begin{aligned}
& 1+(1+A-B+c(1+B))(1-A+B+c(1-B))-\left(\frac{(1+a)\left(1-B^{2}\right)}{A-B}-B\right)^{2} \\
& \geq 2\left|1-B(A-B)+\left(2 B-(A-B)\left(1+B^{2}\right)\right) \frac{1+a}{A-B}+c\left(1-B^{2}\right)\left(1+\frac{B(1+a)}{A-B}\right)\right|
\end{aligned}
$$

when

$$
\left|1-B(A-B)+\left(2 B-(A-B)\left(1+B^{2}\right)\right) \frac{1+a}{A-B}+c\left(1-B^{2}\right)\left(1+\frac{B(1+a)}{A-B}\right)\right| \geq 1 .
$$

If $0 \notin \Phi^{\prime}(\mathbb{D}), 0 \notin \Phi^{\prime \prime}(\mathbb{D})$, then

$$
1+\frac{z \Phi^{\prime \prime}(a ; c, z)}{\Phi^{\prime}(a ; c, z)} \prec \frac{1+A z}{1+B z} .
$$

Proof. For convenience, write $\Phi(z):=\Phi(a ; c ; z)$, and define an analytic function $p: \mathbb{D} \rightarrow \mathbb{C}$ by

$$
p(z):=\frac{(A-B) \Phi^{\prime}(z)+(1-B) z \Phi^{\prime \prime}(z)}{(A-B) \Phi^{\prime}(z)-(1+B) z \Phi^{\prime \prime}(z)}
$$

Then

$$
\frac{z \Phi^{\prime \prime}(z)}{\Phi^{\prime}(z)}=\frac{(A-B)(p(z)-1)}{(p(z)+1)+B(p(z)-1)}
$$

and

$$
\begin{gathered}
\frac{z^{2} \Phi^{\prime \prime \prime}(z)+z \Phi^{\prime \prime}(z)}{z \Phi^{\prime \prime}(z)}-\frac{z \Phi^{\prime \prime}(z)}{\Phi^{\prime}(z)}=\frac{z p^{\prime}(z)}{(p(z)-1)}-\frac{(1+B) z p^{\prime}(z)}{(p(z)+1)+B(p(z)-1)} \\
=\frac{z p^{\prime}(z)((p(z)+1)+B(p(z)-1)-(1+B)(p(z)-1))}{(p(z)-1)((p(z)+1)+B(p(z)-1))} .
\end{gathered}
$$


Thus

$$
\frac{z \Phi^{\prime \prime \prime}(z)}{\Phi^{\prime \prime}(z)}=\frac{2 z p^{\prime}(z)}{(p(z)-1)((p(z)+1)+B(p(z)-1))}-1+\frac{z \Phi^{\prime \prime}(z)}{\Phi^{\prime}(z)}
$$

and together with (4.6) imply that

$$
\begin{aligned}
\left(\frac{z \Phi^{\prime \prime \prime}(z)}{\Phi^{\prime \prime}(z)}\right)\left(\frac{z \Phi^{\prime \prime}(z)}{\Phi^{\prime}(z)}\right) & =\frac{2(A-B)(p(z)-1) z p^{\prime}(z)}{(p(z)-1)((p(z)+1)+B(p(z)-1))^{2}} \\
& -\frac{(A-B)(p(z)-1)}{(p(z)+1)+B(p(z)-1)}+\frac{(A-B)^{2}(p(z)-1)^{2}}{((p(z)+1)+B(p(z)-1))^{2}}
\end{aligned}
$$

A differentiation of (1.2) leads to

$$
z \Phi^{\prime \prime \prime}(z)+(c-z+1) \Phi^{\prime \prime}(z)-(1+a) \Phi^{\prime}(z)=0 .
$$

It follows that

$$
\left(\frac{z \Phi^{\prime \prime \prime}(z)}{\Phi^{\prime \prime}(z)}\right)\left(\frac{z \Phi^{\prime \prime}(z)}{\Phi^{\prime}(z)}\right)+(c-z+1) \frac{z \Phi^{\prime \prime}(z)}{\Phi^{\prime}(z)}-(1+a) z=0 .
$$

Using (4.6) and (4.7), (4.8) yields

$$
\begin{aligned}
& \frac{2(A-B) z p^{\prime}(z)}{((p(z)+1)+B(p(z)-1))^{2}}+\frac{(A-B)^{2}(p(z)-1)^{2}}{((p(z)+1)+B(p(z)-1))^{2}} \\
& +\frac{(A-B)(p(z)-1)(c-z)}{(p(z)+1)+B(p(z)-1)}-(1+a) z=0,
\end{aligned}
$$

that is,

$$
\begin{aligned}
z p^{\prime}(z)+ & \left(\frac{(A-B)}{2}+\frac{(c-z)(1+B)}{2}-\frac{(1+a) z(1+B)^{2}}{2(A-B)}\right)(p(z))^{2} \\
- & \left(A-B+(c-z) B+\frac{(1+a)\left(1-B^{2}\right)}{(A-B)} z\right) p(z)+\left(\frac{(A-B)}{2}+\right. \\
& \frac{(c-z)(-1+B)}{2}-\frac{(1+a) z(1-B)^{2}}{2(A-B)}=0 .
\end{aligned}
$$

Denote by $\Psi\left(p(z), z p^{\prime}(z), z\right):=z p^{\prime}(z)+F_{1}(p(z))^{2}+F_{2} p(z)+F_{3}$, where

$$
\begin{aligned}
& F_{1}=\frac{(A-B)}{2}+\frac{(c-z)(1+B)}{2}-\frac{(1+a) z(1+B)^{2}}{2(A-B)}, \\
& F_{2}=-(A-B)-(c-z) B-\frac{(1+a)\left(1-B^{2}\right)}{(A-B)} z, \\
& F_{3}=\frac{(A-B)}{2}+\frac{(c-z)(-1+B)}{2}-\frac{(1+a) z(1-B)^{2}}{2(A-B)} .
\end{aligned}
$$


With $\Omega=\{0\},(4.9)$ yields $\Psi\left(p(z), z p^{\prime}(z), z\right) \in \Omega$. Now with $z=x+i y \in \mathbb{D}$, let

$$
\begin{aligned}
G_{1}:=\operatorname{Re}\left(F_{1}\right) & =\left(\frac{A-B}{2}+\frac{(c-x)(1+B)}{2}-\frac{(1+a) x(1+B)^{2}}{2(A-B)}\right) . \\
& =\frac{1}{2}\left(A-B+c(1+B)-x(1+B)\left(1+\frac{(1+a)(1+B)}{A-B}\right)\right), \\
G_{2}:=\operatorname{Re}\left(i F_{2}\right) & =-y B+\frac{(1+a)\left(1-B^{2}\right)}{(A-B)} y, \\
G_{3}:=\operatorname{Re}\left(F_{3}\right) & =\left(\frac{A-B}{2}+\frac{(c-x)(-1+B)}{2}-\frac{(1+a) x(1-B)^{2}}{2(A-B)}\right) \\
& =\frac{1}{2}\left(A-B-c(1-B)+x(1-B)\left(1-\frac{(1+a)(1-B)}{A-B}\right)\right) .
\end{aligned}
$$

For $\sigma \leq-\left(1+\rho^{2}\right) / 2, \rho \in \mathbb{R}$,

$$
\begin{aligned}
\operatorname{Re} \Psi(i \rho, \sigma, z) & =\sigma-G_{1} \rho^{2}+G_{2} \rho+G_{3} \\
& \leq-\frac{1}{2}\left(\left(1+2 G_{1}\right) \rho^{2}-2 G_{2} \rho-2 G_{3}+1\right):=Q(\rho) .
\end{aligned}
$$

Note that condition (4.1) implies $\left(1+2 G_{1}\right) / 2>0$. In this case, $Q$ has a maximum at $\rho=G_{2} /\left(1+2 G_{1}\right)$. Thus $Q(\rho)<0$ for all real $\rho$ provided

$$
G_{2}^{2} \leq\left(1+2 G_{1}\right)\left(1-2 G_{3}\right), \quad|x|,|y|<1 .
$$

Since $y^{2}<1-x^{2}$, it is left to show that

$$
\begin{aligned}
& \left(\frac{(1+a)\left(1-B^{2}\right)}{A-B}-B\right)^{2}\left(1-x^{2}\right) \\
& \leq\left(1+A-B+c(1+B)-x(1+B)\left(1+\frac{(1+a)(1+B)}{A-B}\right)\right) \\
& \quad \times\left(1-A+B+c(1-B)-x(1-B)\left(1-\frac{(1+a)(1-B)}{A-B}\right)\right)
\end{aligned}
$$

$|x|<1$. The above inequality is equivalent to

$$
H(x):=x^{2}-2 h_{2}(A, B) x+h_{3}(A, B) \geq 0,
$$

where

$$
\begin{aligned}
\begin{aligned}
h_{2}(A, B)=1-B A+B^{2}+\frac{\left(2 B-(A-B)\left(1+B^{2}\right)\right)(1+a)}{A-B} \\
+\left(1+\frac{B(1+a)}{A-B}\right)\left(1-B^{2}\right) c,
\end{aligned} \\
\begin{aligned}
h_{3}(A, B)=(1+A-B+c(1+B))(1-A+B & +c(1-B)) \\
& -\left(\frac{(1+a)\left(1-B^{2}\right)}{A-B}-B\right)^{2} .
\end{aligned}
\end{aligned}
$$


To establish (4.10), first consider the case when (4.3) holds. Then $\left|h_{2}\right| \leq 1$, and hence $H^{\prime}(x)=0$ at $x=h_{2} \in(-1,1)$. Since $H^{\prime \prime}(x)>0, H$ has a minimum at $x=h_{2}$ and from (4.2), it follows that

$$
H(x) \geq H\left(h_{2}\right)=h_{2}^{2}-2 h_{2}^{2}+h_{3}=-h_{2}^{2}+h_{3} \geq 0 .
$$

Now consider the case when (4.5) holds. In this case $\left|h_{2}\right| \geq 1$, and $H^{\prime}(x)=$ $2\left(x-h_{2}\right) \leq 2\left(1-h_{2}\right) \leq 0$. Hence $H$ is decreasing. From (4.4), it is evident that

$$
\begin{aligned}
H(x) \geq H(1) & =1-2 h_{2}+h_{3} \\
& \geq 1-2\left|h_{2}\right|+h_{3} \geq 0 .
\end{aligned}
$$

Thus $\Psi$ satisfies the hypothesis of Lemma 1.1, and hence $\operatorname{Re} p(z)>0$, or equivalently

$$
\frac{(A-B) \Phi^{\prime}+(1-B) z \Phi^{\prime \prime}}{(A-B) \Phi^{\prime}-(1+B) z \Phi^{\prime \prime}} \prec \frac{1+z}{1-z} .
$$

By definition of subordination, there exists an analytic self-map $w$ of $\mathbb{D}$ with $w(0)=0$ and

$$
\frac{(A-B) \Phi^{\prime}(z)+(1-B) z \Phi^{\prime \prime}(z)}{(A-B) \Phi^{\prime}(z)-(1+B) z \Phi^{\prime \prime}(z)}=\frac{1+w(z)}{1-w(z)}
$$

A simple computation shows that

$$
1+\frac{z \Phi^{\prime \prime}(z)}{\Phi^{\prime}(z)}=\frac{1+A w(z)}{1+B w(z)}
$$

and hence

$$
1+\frac{z \Phi^{\prime \prime}(z)}{\Phi^{\prime}(z)} \prec \frac{1+A z}{1+B z} .
$$

The relation (1.3) also shows that

$$
\frac{z(z \Phi(a ; c ; z))^{\prime}}{z \Phi(a ; c ; z)}=1+\frac{z \Phi^{\prime \prime}(a-1 ; c-1 ; z)}{\Phi^{\prime}(a-1 ; c-1 ; z)} .
$$

Together with Theorem 4.1, it immediately yields the following result for $z \Phi(a ; c ; z) \in \mathcal{S}^{*}[A, B]$.

Theorem 4.2. Let $a$ and $c$ be real numbers such that $(A-B) \Phi^{\prime}(a-1 ; c-1, z) \neq$ $(1+B) z \Phi^{\prime \prime}(a-1 ; c-1 ; z),-1 \leq B<A \leq 1$. Suppose

$$
c(1+B) \geq(1+B)\left|1+\frac{a(1+B)}{A-B}\right|-(A-2 B) .
$$

Further let $A, B, c$ and a satisfy either

$$
\left(1-B(A-B)+\left(2 B-(A-B)\left(1+B^{2}\right)\right) \frac{a}{A-B}+(c-1)\left(1-B^{2}\right)\left(1+\frac{B a}{A-B}\right)\right)^{2}
$$




$$
\leq(A-2 B+c(1+B))(2 B-A+c(1-B))-\left(\frac{a\left(1-B^{2}\right)}{A-B}-B\right)^{2}
$$

whenever

$$
\begin{aligned}
& \mid 1-B(A-B)+\left(2 B-(A-B)\left(1+B^{2}\right)\right) \frac{a}{A-B}+ \\
& \quad(c-1)\left(1-B^{2}\right)\left(1+\frac{B a}{A-B}\right) \mid \leq 1,
\end{aligned}
$$

or

$$
\begin{aligned}
1+(A-2 B+ & +c(1+B))(2 B-A+c(1-B))-\left(\frac{a\left(1-B^{2}\right)}{A-B}-B\right)^{2} \\
\geq 2 \mid 1-B(A-B)+\left(2 B-(A-B)\left(1+B^{2}\right)\right) \frac{a}{A-B}+ & \left(1+\frac{B a}{A-B}\right)\left(1-B^{2}\right)(c-1) \mid
\end{aligned}
$$

when

$$
\begin{aligned}
& \mid 1-B(A-B)+\left(2 B-(A-B)\left(1+B^{2}\right)\right) \frac{a}{A-B}+ \\
& \qquad\left(1+\frac{B a}{A-B}\right)\left(1-B^{2}\right)(c-1) \mid \geq 1 .
\end{aligned}
$$

Then $z \Phi(a ; c ; z) \in \mathcal{S}^{*}[A, B]$.

Remark 4.1. Choosing $A=1-2 \beta, \beta \in[0,1)$, and $B=-1$, then Theorem 4.1 gives [10, Theorem 5.1, p. 88], while Theorem 4.2 gives [10, Theorem 5.3, p. 88]. For $a=1$, $c=1+\delta, \delta \geq 1$, Theorem 4.1 gives sufficient conditions for

$$
\Phi(1 ; 1+\delta ; z)=\delta \int_{0}^{1}(1-t)^{\delta-1} e^{t z} d t
$$

to be Janowski convex. This is a generalization of a result in [10, Corollary 5.8, p. 89].

Writing $f(z)=z \Phi(a ; c ; z)$, and $g(z)=f\left(z^{2}\right) / z$, it follows that

$$
\frac{z g^{\prime}(z)}{g(z)}=2 \frac{z^{2} f^{\prime}\left(z^{2}\right)}{f\left(z^{2}\right)}-1
$$

Now (4.16) and Theorem 4.2 immediately yield the following result.

Corollary 4.1. Let $a$ and $c$ be real numbers such that $(A-B) \Phi^{\prime}(a+1 ; c+1 ; z) \neq$ $(1+B) z \Phi^{\prime \prime}(a+1 ; c+1 ; z)$ and (4.11) holds for $-1 \leq B<2 A-B \leq 1$. Further let $A, B, c$ and a satisfy either (4.12) when (4.13) holds, or (4.14) when (4.15) holds. Then $z \Phi\left(a ; c ; z^{2}\right) \in \mathcal{S}^{*}[2 A-B, B]$. 
Remark 4.2. Particular choice of $A$ and $B$ yield the following ramifications.

(i) For $A=1-2 \beta$ and $B=-1$, Corollary 4.1 reduces to [10, Corollary 5.13, p. 93].

(ii) For $a=1 / 2$, Corollary 4.1 is useful in obtaining conditions for the generalized error function $z \Phi(1 / 2 ; c, z) \in \mathcal{S}^{*}[2 A-B, B]$.

Acknowledgment. The work presented here was supported by a FRGS and RU research grants from Universiti Sains Malaysia. The authors are thankful to the referee for the insightful comments that helped improve the clarity of this manuscript.

\section{References}

[1] A. P. Acharya, Univalence criteria for analytic functions and applications to hypergeometric functions, Ph.D diss., University of Würzburg, 1997.

[2] R. M. Ali, V. Ravichandran and N. Seenivasagan, Sufficient conditions for Janowski starlikeness, Int. J. Math. Math. Sci. 2007, Art. ID 62925, 7 pp.

[3] R. M. Ali, R. Chandrashekar and V. Ravichandran, Janowski starlikeness for a class of analytic functions, Appl. Math. Lett. 24 (2011), no. 4, 501-505.

[4] W. W. Bell, Special functions for scientists and engineers, D. Van Nostrand Co., Ltd., London, 1968.

[5] A. W. Goodman, Univalent functions. Vol. I E II, Mariner, Tampa, FL, 1983.

[6] W. Janowski, Some extremal problems for certain families of analytic functions. I, Ann. Polon. Math. 28 (1973), 297-326.

[7] S. S. Miller and P. T. Mocanu, Univalence of Gaussian and confluent hypergeometric functions, Proc. Amer. Math. Soc. 110 (1990), no. 2, 333-342.

[8] S. S. Miller and P. T. Mocanu, Differential subordinations and inequalities in the complex plane, J. Differential Equations 67 (1987), no. 2, 199-211.

[9] S. S. Miller and P. T. Mocanu, Differential subordinations, Monographs and Textbooks in Pure and Applied Mathematics, 225, Dekker, New York, 2000.

[10] S. Ponnusamy and M. Vuorinen, Univalence and convexity properties for confluent hypergeometric functions, Complex Variables Theory Appl. 36 (1998), no. 1, 73-97. 
[11] St. Ruscheweyh and V. Singh, On the order of starlikeness of hypergeometric functions, J. Math. Anal. Appl. 113 (1986), no. 1, 1-11.

[12] N. M. Temme, Special functions, A Wiley-Interscience Publication, Wiley, New York, 1996.

School of Mathematical Sciences, Universiti Sains Malaysia, 11800 USM Penang Malaysia

email:rosihan@usm.my

Department of Mathematics, King Faisal University,

Ahsaa 31982, Saudi Arabia

email:smondal@kfu.edu.sa

Department of Mathematics, University of Delhi,

Delhi 110007, India

email:vravi@maths.du.ac.in 